\title{
Evaluation of injectorless quantum cascade lasers by combining XRD- and laser-characterisation
}

\author{
Christian Grasse*, Simeon Katz, Gerhard Böhm, Augustinas Vizbaras, \\ Ralf Meyer, Markus-Christian Amann \\ Walter Schottky Institut, Technische Universität München, Am Coulombwall 3, Garching 85748, Germany
}

\section{A R T I C L E I N F O}

Available online 4 November 2010

\section{Keywords:}

A3. Laserepitaxy

B1. Arsenates

B1. Phosphides

B2. Semiconducting III/V materials

B3. Infrared devices

B3. Laserdiodes

\begin{abstract}
A B S T R A C T
We present a wavelength prediction procedure on the basis of X-ray measurements and simulations for InP-based injectorless quantum cascade laser (QCL) devices. These lasers show excellent performance in the mid-infrared wavelength range, but are very sensitive to growth deviations, which cause strong wavelength shifts and are a serious obstacle for applications like gas sensing. However, by XRD-simulations of the active region, which consists of InAs, AlAs, GaInAs and AlInAs, the thicknesses and compositions can be extracted and are used as input values for bandstructure calculations, so that a prediction for the resonance energy of the laser transition can be obtained. With this technique a wavelength evaluation of injectorless QCLs with $3 \%$ accuracy could be accomplished, which is an essential improvement in applications like gas sensing.
\end{abstract}

(c) 2010 Elsevier B.V. All rights reserved.

\section{Introduction}

Recent progress in applications like gas sensing has strongly increased the demand on high performance quantum cascade lasers (QCL) [1]. Since these unipolar devices are based on intraband transitions, a wavelength range beyond $3 \mu \mathrm{m}$, and therefore strong characteristic absorption lines of many molecules, are accessible with these devices, with the advantage of continuous wave room temperature operation. This gives them an auspicious market position compared to the standard lead salt diodes, which need cryogenic cooling [1].

From the first realization of this laser concept in 1994 [2] steady improvements resulted in injectorless QCLs based on InP, which avoid the optically inert injector section normally needed for carrier cascading without affecting $c w$-operation [3]. The particular advantage of the injectorless design lies in increased overlap of the waveguide mode with the active sections and reduced internal losses. Thus, injectorless QCL showed the lowest (pulsed) threshold densities for all types of QCL and are predicted to achieve the best power conversion efficiency [3,4].

As the MBE grown active region consists of very thin layers (0.5-5 nm), growth deviations due to small shutter opening times (shortest $1 \mathrm{~s}$ ) have a strong influence on device performance. Thus, wavelength shifts, which would be critical for gas sensing, have to be prevented.

\footnotetext{
* Corresponding author. Tel.: +4989 28912789

E-mail address: grasse@wsi.tum.de (C. Grasse).
}

To investigate the sensitivity of injectorless QCLs against growth deviations and to establish a method to predict the laser wavelength, several QCL structures identical in design have been grown by MBE and were processed. A comparison of the different laser wavelengths from these devices showed a maximum deviation of around $1 \mu \mathrm{m}$ corresponding to $30 \mathrm{meV}$ ( $17 \%$ of emission energy) and will be discussed on the basis of X-ray diffraction measurements (XRD) and bandstructure calculations.

\section{Experimental procedure}

The structures were grown with a Varian Mod Gen II MBE system equipped with two aluminium, one gallium, indium and a silicon evaporation cell together with two valved cracker cells for phosphorus and arsenic. The test structures consist of a four material active region [4] with 60 stages (except sample M4206, which had 65 stages), schematically sketched in Fig. 1, sandwiched between two $520 \mathrm{~nm}$ thick silicon-doped GaInAs confinement layers and followed by a silicon-doped $2.5 \mu \mathrm{m} \mathrm{InP}$ and $1.0 \mu \mathrm{m}$ GaInAs top-cladding layer grown on (low) $n$-doped InP substrates. During growth of the active region always both aluminium cells were used at the same time to achieve a sufficient growth rate. After cleaving, the wafers were partly processed into MCRW-lasers, whereas one piece served as X-ray diffraction (XRD) sample (in this case the cladding layer was selectively etched away). XRD-measurements were carried out with a STOE single crystal diffractometer and emission-spectra were recorded using a Vertex 70 Fourier transform infrared (FTIR) spectrometer made by Bruker. 


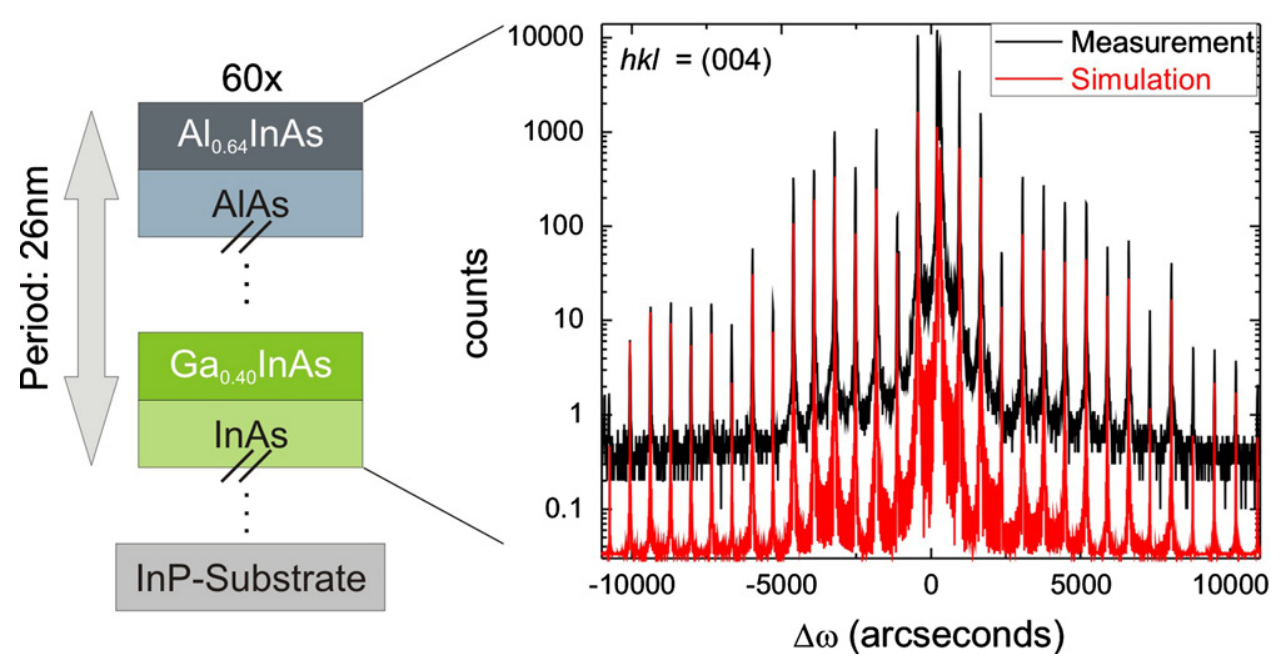

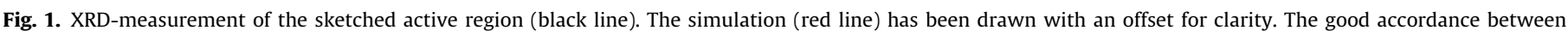

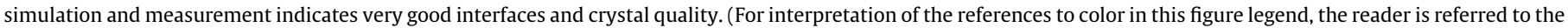
web version of this article.)

For XRD-simulations the "Bede Rads 4.0" software (now Jordan Valley) was used.

\subsection{QCL-simulation}

The active region of all samples is equal to the layer structure

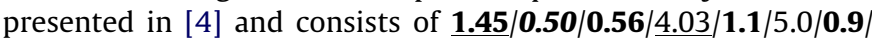
2.6/0.51/2.6/1.0/2.1/1.0/2.66, with $\mathbf{A l}_{\mathbf{0 . 6 3 5}}$ InAs in bold, AlAs in bold italic, $\mathrm{Ga}_{0.4}$ InAs in regular and InAs in italic style. Since the device has 60 stages each with 14 layers, a reduction of simulation parameters is essential for meaningful results. This can be achieved by considering that only three variables, aluminium- (from both cells), indium- and gallium-flux, are involved in the structure. Thus, also the simulation should be reduced to three variables. Therefore all simulations have been carried out under the following conditions:

1. No drift of the cell flux and hence, of the compositions and thicknesses during growth has been assumed, which is reasonable, since the whole active region is only $1.6 \mu \mathrm{m}$ thick and all cells are kept at constant temperature and flux.

2. Only the two underlined layers have been released as simulation parameters, which means that the simulation program uses the composition and thickness of only those two layers to fit the measurement. Thus, the remaining AlInAs and GaInAs layers have the same composition as layers 1 and 4, respectively. The thickness of those layers follows from the simulated AlInAs and GaInAs growth rate (thickness divided by the corresponding growth time) multiplied with the growth time of the individual layer. Finally, in the case of AlAs and InAs growth for the thickness $d$ follows (here e.g. AlAs):

$d_{\mathrm{AlAs}}=x_{\mathrm{Al}} \frac{d_{\mathrm{Al}_{\mathrm{Al}_{\mathrm{Al}}} I n_{1-\chi_{\mathrm{Al}}} \mathrm{As}}}{t_{\mathrm{Al}_{\mathrm{Al}_{\mathrm{Al}}} I n_{1-x_{\mathrm{Al}}} \mathrm{As}}} \frac{a_{p m, \mathrm{AlAs}}}{a_{p m, \mathrm{Al}_{\mathrm{Al}} I n_{1-x_{\mathrm{Al}}} \mathrm{As}}} t_{\mathrm{AlAs}}$

The first part of the equation is given by layer 1 , followed by the ratio of the pseudomorphic lattice constants of AlAs and AlInAs to correct the growth rate by influence of the strained unit cell. This is equal to aluminium-flux times the pseudomorphic lattice constant of the growing layer and therefore growth rate. Multiplication with the growth time then leads to the layer thickness. In case of InAs layer 4 instead of 1 was deployed.

Finally the reduction to three variables is accomplished by matching the indium-flux from layer 1 and 4 during the iterations.
In this way reproducible simulations could be achieved, which also fit well to the measurement as shown in Fig. 1.

\section{Results and discussion}

The procedure for analyzing the different devices was as follows: first an XRD-measurement was done and from the simulation of this measurement the period length and compositions were extracted. With this information bandstructure calculations were carried out using the "nextnano" software [5]. In this way, a minimum and maximum value for the expected laser wavelength could be calculated, which is defined by lasing as designed (minimum, $\lambda_{\text {min }}$ ) and an additional lasing condition with lower gain using a resonance below the design field $\left(\lambda_{\max }\right.$, see Fig. 2), which means that this transition energy is always lower than the designed transition. This is a special feature of injectorless QCLs, since compared to injectorbased devices, injectorless designs allow current flow only for two resonance conditions, which are energetically separated by the energy of one phonon. Either the ground state or the second state is in resonance with the subsequent upper laser level, strongly influencing the emission energy, as the transition energy is a combination of quantization energy defined by the well width and the energetical shift due to the applied field (diagonal laser transition, see Fig. 2).

This behaviour is illustrated in Fig. 3, where the emission spectrum depending on the current density has been collected. Increasing the current density results in an increased field, which in turn causes a blueshift of the wavelength towards the design transition $\lambda_{\min }$ (shown as dashed line in Fig. 3). For the threshold wavelength, on the other hand, an intermediate state between both laser transitions $\left(\lambda_{\min } \mu \lambda_{\max }\right.$ ) occurs, which is then dominated by the designed resonance emission with increasing current. In addition, this shift of states in alignment was also observed in the current dependence of gain coefficient, which confirms this theory [6,7].

A comparison between simulated and measured laser wavelengths is given in Table 1 . For all four devices the measured wavelength at threshold $\left(\lambda_{\text {th }}\right)$ lies between the simulated extrema, whereas the emission wavelength at the rollover of the laser $\left(\lambda_{\text {rollover }}\right)$ is close to the designed resonance $\left(\lambda_{\min }\right)$ as expected. This indicates that a prediction of the emission wavelength and an estimation of the spectral range of operation are possible in this manner. 
Fig. 4 emphasizes this conclusion: all samples show a linear dependency between measured laser wavelength at rollover and average pseudomorphic strain $(\Delta a / a$ see Table 1$)$, which is indicated by the linear fit (dashed line) in Fig. 4 . This can be understood on the basis that higher tensile pseudomorphic strain means mainly higher aluminium content, yielding higher barriers and therefore increased quantization energy, which results in a decreased emission wavelength. Comparing $\lambda_{\text {rollover }}$ with a linear fit of the simulated resonance transition $\lambda_{\min }$ from all samples with 60 stages (dotted line in Fig. 4) yields that this tendency is also confirmed by the simulations with a maximum deviation of $3 \%$. However, sample M4206 with 65 stages, showed a strong shift of the threshold wavelength, which is completely dominated by

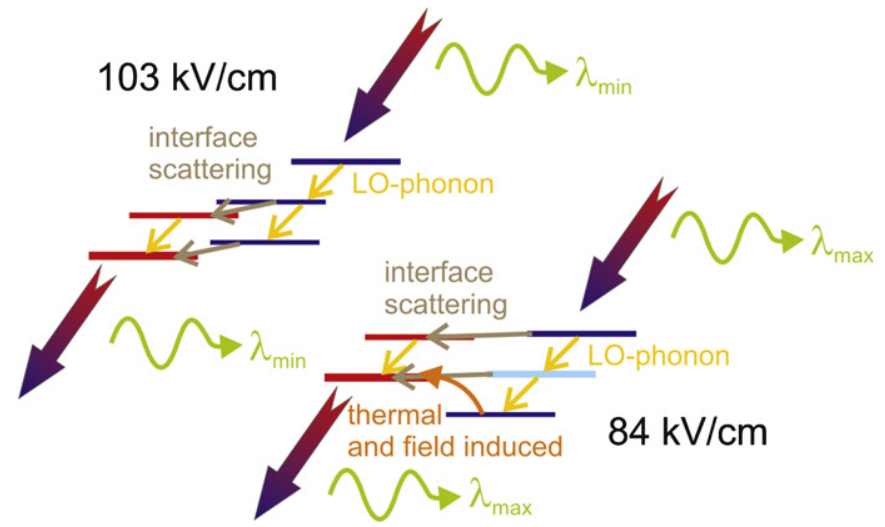

Fig. 2. Resonance schematics for design (left) and below design field (right).

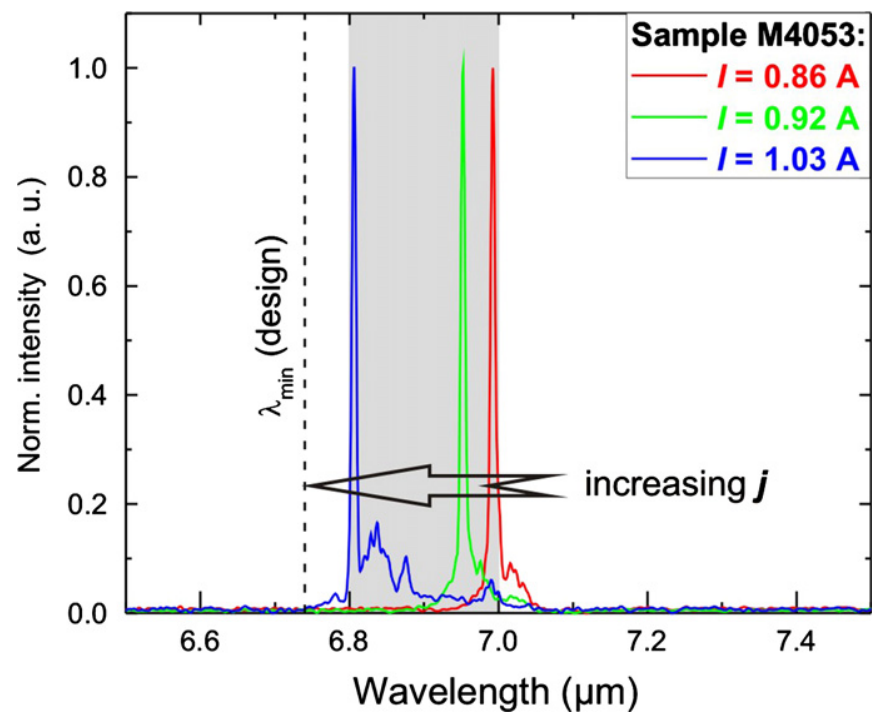

Fig. 3. The emission spectrum over dynamic range is shown for sample M4053. The grey area shows the wavelength shift to designed transition (dashed line) with increase in current. second state emission (corresponding to $\lambda_{\max }$ ), whereas the deviation between $\lambda_{\text {rollover }}$ and the fit prediction is just $3 \%$. Since the number of stages was also taken into account by the simulation, it can be concluded that a prediction for $\lambda_{\text {th }}$ is more complex than for $\lambda_{\text {rollover }}$. The influence of varying doping levels and total losses could explain this emission characteristic, which can be seen as a limit for XRD based QCL characterisation.

Nevertheless, a forecast for the minimum laser wavelength of injectorless QCLs on the basis of XRD characterisation is possible, whereas for the maximum and, hence, threshold wavelength only an upper limit, the second state transition, is predictable, when design changes like increased number of stages are included into the evaluation. However, since higher transition energies are accessible only by increasing the current density (shown in Fig. 4), a blueshift with respect to the desired wavelength cannot be compensated. Therefore a wavelength prediction as discussed saves processing time and allows growth correction for the next run to adjust the wavelength needed for applications like gas sensing.

\section{Conclusions}

Injectorless QCLs are promising as high power mid-infrared sources, but very sensitive to growth deviations, which cause a strong shift in wavelength and are, hence, critical for applications like gas sensing. To achieve a laser wavelength prediction after growth, XRD- and wavelength-measurements of different devices, identical in design, have been correlated with bandstructure calculations. In this way a forecast for the wavelength range of a device is possible before processing and also corrections for the next growth can be rendered.

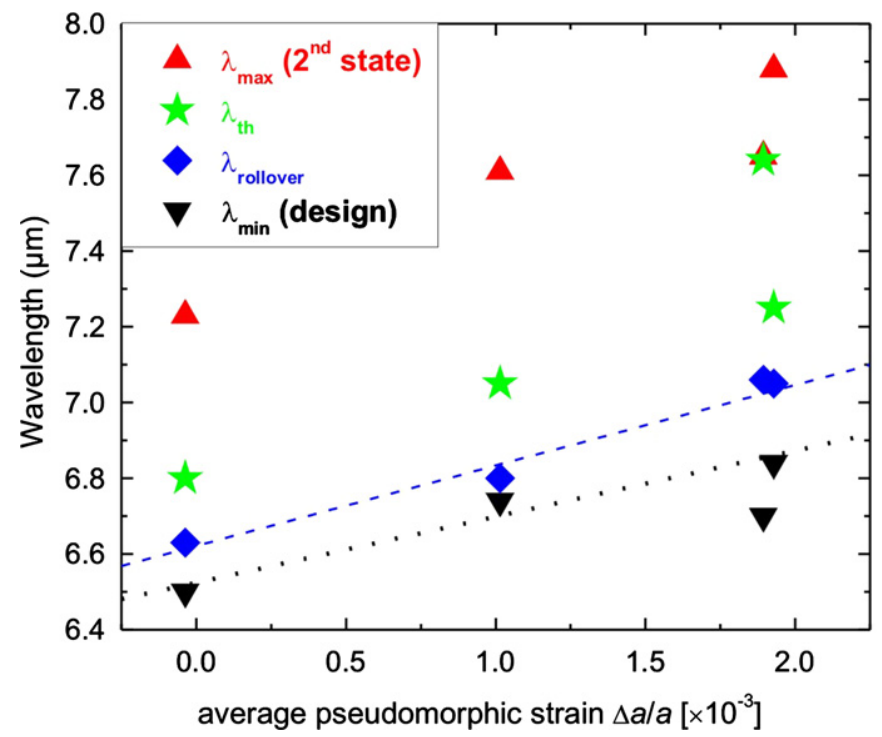

Fig. 4. Overview of measured laser wavelengths $\left(\lambda_{\text {th }}, \lambda_{\text {rollover }}\right)$ and simulated resonance states (see Table 1) against average pseudomorphic strain of the active region. Linear fits of $\lambda_{\text {rollover }}$ and $\lambda_{\text {min }}$ from all samples with 60 stages are shown as dashed and dotted lines, respectively.

Table 1

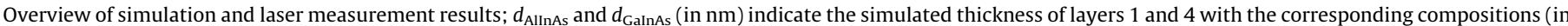
$\%)$. The compositions are defined as $\mathrm{Al}_{x(\mathrm{Al})} \mathrm{In}_{1-x(\mathrm{Al})} \mathrm{As}$ and $\mathrm{Ga}_{x(\mathrm{Ga})} \mathrm{In}_{1-x(\mathrm{Ga})} \mathrm{As}$, respectively. All lasers have the same length $(4 \mathrm{~mm})$ and width $(26 \mu \mathrm{m})$.

\begin{tabular}{|c|c|c|c|c|c|c|c|c|c|c|}
\hline Sample ID & $d_{\text {AlInAs }}(n m)$ & $x(\mathrm{Al})(\%)$ & $d_{\text {GaInAs }}(\mathrm{nm})$ & $x(\mathrm{Ga})(\%)$ & $d_{\mathrm{SL}}(\mathrm{nm})$ & $\Delta a_{\mathrm{pm}} / a$ & $\lambda_{\min }($ design $)(\mu \mathrm{m})$ & $\lambda_{\max }(2 \mathrm{nd}$ state $)(\mu \mathrm{m})$ & $\lambda_{\text {th }}(\mu \mathrm{m})$ & $\lambda_{\text {rollover }}(\mu \mathrm{m})$ \\
\hline M3856 & 1.48 & 64.4 & 3.97 & 39.4 & 26.0 & $1.9 \times 10^{-3}$ & 6.84 & 7.88 & 7.25 & 7.05 \\
\hline M4053 & 1.48 & 65.1 & 3.93 & 40.1 & 25.8 & $1.0 \times 10^{-3}$ & 6.74 & 7.61 & 7.05 & 6.80 \\
\hline M4206 & 1.49 & 64.9 & 3.96 & 39.2 & 25.9 & $1.9 \times 10^{-3}$ & 6.70 & 7.65 & 7.64 & 7.06 \\
\hline M4522 & 1.55 & 66.1 & 4.06 & 40.7 & 26.7 & $-3.6 \times 10^{-5}$ & 6.50 & 7.23 & 6.80 & 6.63 \\
\hline
\end{tabular}


This is essential for future applications and can be carried forward to even more complicated devices like THz-QCLs, which contain critical fabrication steps like overgrowths [8].

\section{Acknowledgment}

This work was partly supported by the Excellence Cluster "Nanosystems Initiative Munich (NIM)".

\section{References}

[1] J. Shorter, et al., IEEE Sensors J. 10 (2010) 76-84.

[2] J. Faist, et al., Science 264 (1994) 553.

[3] S. Katz, et al., Semicond. Sci. Technol. 24 (2009) 122001.

[4] S. Katz, et al., Electron. Lett. 44 (2008) 580-581.

[5] see 〈www.nextnano.de $\rangle$.

[6] E. Benveniste, et al., Appl. Phys. Lett. 94 (2009) 081110.

[7] S. Katz et al., Nonlinear gain behaviour in injectorless quantum cascade lasers, Opt. Eng., in press (Reference No. 003011JOE).

[8] M.A. Belkin, et al., Nat. Photon 1 (2007) 288. 